\title{
Stimulus stringing by pigeons: Conditional strings
}

\author{
W. KIRK RICHARDSON and JEFFREY A. KRESCH \\ Georgia State University, Atlanta, Georgia
}

\begin{abstract}
Pigeons were trained to produce one serial list in the presence of a green background cue and another serial list in the presence of a red background cue when the items for both serial lists were presented on each trial. This demonstrated a combination of serial learning and conditional discrimination learning not previously shown in pigeons. Specifically, when presented with four geometric forms, A B C D, in random locations of a five-key display, the pigeons learned to peck A B C when the background was green and A B D when the background was red. Accuracy on the conditional string ranged from $73 \%$ to $85 \%$. Transfer tests using different locations of the stimuli on the keys showed positive transfer, thus ruling out learning of specific locations as the basis of the accurate performance. Above-chance performance was maintained when the conditional colors were presented only on the key that did not contain one of the serial stimuli. The results are interpreted in terms of a chaining model that postulates that the sequential selections were controlled by cues produced by both onset of the trial and prior selections within the trial.
\end{abstract}

Serial learning has long been a research topic, especially in the literature on humans. Currently, there are a number of paradigms being used to study serial learning in lower animals. Sands and Wright (1980) have demonstrated that rhesus monkeys have a remarkable capacity to learn a long serial list. Capaldi's theory of partial reinforcement has been extended to the learning of monotonic and nonmonotonic series of quantities of food rewards (Capaldi, Verry, \& Davidson, 1980). Hulse (1978) has also carried out extensive studies of serial pattern learning for series of food quantities. Studies of spatial memory in the radial arm maze (Olton, 1978) can also be viewed as using a serial task.

The pervasiveness of serial behavior means that each organism generally engages in different behavior sequences at different times. The particular sequence active at a given time would presumably be due to some stimulus, either discriminative or eliciting, that controls the behavioral sequence. However, the paradigms used to study serial behavior generally involve only one such sequence for a given subject. When different sequences are studied, they are usually given to different groups of animals.

The present study extends a paradigm used in our laboratory to study single sequences in pigeons (Richardson \& Bittner, 1982; Richardson \& Warzak,

This study was based on a thesis submitted to the Department of Psychology, Georgia State University, in partial fulfillment of the requirements for the MA degree by the second author. We wish to thank the staff of the Georgia State University Computer Center for technical assistance. Reprints may be obtained from W. Kirk Richardson, Psychology Department, University Plaza, Atlanta, Georgia 30303.
1981) to the study of performance by individual organisms on two different sequences. In our earlier work, the serial items were colors projected on response keys. Each color was assigned to a serial position in the stimulus string (sequence). If we called the first color A, the second color B, etc., then the task required the subject to peck $A$ B C D in that order in the four-stimulus string condition. The location of the colors on the keys was varied randomly from one correct trial to the next, and a between-trials correction procedure was used to insure that the subject learned each array. Correctly selected colors changed from dim to bright for the remainder of the trial. Thus, a correctly selected stimulus was explicitly "tagged." The trial terminated after a correct sequence or one incorrect selection.

The present study extended the earlier work in two ways. First, the items consisted of lexigrams (geometric drawings) projected as white illuminated lines. All earlier work had used colors for the serial items. Because color was known to be a very salient cue for pigeons, we wished to see if pigeons could learn a string when the stimuli seemed to have no special salience. The second, and more important, extension was the requirement that the pigeon learn two strings. The elements for both strings were provided, and an additional cue indicated whether string 1 or string 2 was correct on a given trial. The additional cue was one of two colors, red or green, projected as the background color for the lexigrams. The task was thus a conditional discrimination, in which the conditional behavior was the selection of a stimulus string. Because the correct sequence of lexigrams was indicated by the background color and because there was no physical similarity between the background cues 
and the lexigrams, the task can be referred to as a conditional, symbolic, stimulus-string task.

\section{EXPERIMENT 1}

Experiment 1 of the present study answered two primary questions. First, could pigeons learn a stimulus string when the elements of the string did not have any special salience for pigeons? Second, could pigeons learn to select one of two possible stimuli in the variable serial position of a string when the correct stimulus selection was cued by the value of an environmental cue? If the pigeons learned this task, then more molecular analyses would help to understand the learning processes involved.

\section{Method}

Subjects. The subjects were eight naive racing homing pigeons maintained at $75 \%$ of their free-feeding weights. Three subjects were assigned randomly to Group A (Stimulus Set A), and five subjects were assigned randomly to Group B (Stimulus Set B).

Apparatus. Two test chambers had inside dimensions of $50 \times$ $36 \times 36 \mathrm{~cm}(\mathrm{~L} \times \mathrm{W} \times \mathrm{H})$ and a jeweled light centered on the rear wall $4 \mathrm{~cm}$ from the ceiling. Opposite to the rear wall was an aluminum-alloy false wall with two rows of five $2.5-\mathrm{cm}$-square openings. The top row had its upper edge $7 \mathrm{~cm}$ below the top of the chamber and its left edge $14 \mathrm{~cm}$ from the left wall. The bottom row was $4.5 \mathrm{~cm}$ below the upper row. There was $1.5 \mathrm{~cm}$ between the sides of adjacent openings. A Seientific Prototype food cup was located on the lower left of the front wall, $1 \mathrm{~cm}$ above the floor and $2 \mathrm{~cm}$ from the wall. The food cup could be illuminated by a hooded lamp centered $.5 \mathrm{~cm}$ above the food cup. A 75-dB (re $20 \mathrm{SPL}$ ) white noise and a ventilation fan provided auditory masking.

An Industrial Electronics Engineers Series 10 in-line display cell was located behind each opening. Each display cell contained three colors and nine geometric forms, the same elements used to construct the lexigrams of the Yerkish language (von Glaserfeld, 1977). Two sets of four stimulus cues (lexigrams) were formed from randomly selected pairs of the nine geometric forms (Figure 1). The lexigrams could be projected against background colors of black (i.e., no color), green, or red, and the colorlexigram compound could be illuminated at two intensities, dim ( $4 \mathrm{~V}$ ) and bright (6.5 V), by No. 44 miniature lamps. Transparent Lexan paddles located between the openings and the display cells could be operated by a static force of 5-15 g (.005-.05 N) through an excursion of .5-1.0 mm.

\section{A. Stimulus Set A.}

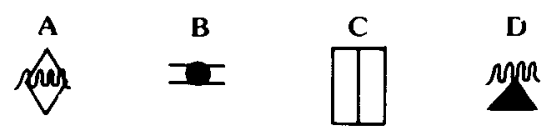

B. Stimulus Set B.

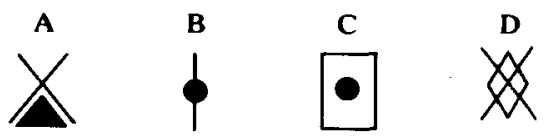

Figure 1. Schematic drawings of the lexigrams used as serial items in the strings.
An Interdata 732 computer controlled the experimental task and data collection.

Procedure. After adaptation and magazine training, the subjects were trained to peck Stimulus $A$ on a black background using the method of shaping by successive approximations. Pecks to Stimulus $A$ (the first stimulus in the sequence) produced food and then trial termination. In all phases of the experiment, any incorrect response produced a .5-sec tone and then trial termination, whereas correct strings produced food. When the subjects were reliably tracking Stimulus A, the two-stimulus sequence (A B) was introduced. When the subjects were reliably pecking the sequence $A B$, the three-stimulus sequence was introduced.

In the three-stimulus condition, there were two groups of three stimuli presented to each subject: A B C and A B D. When A B C was presented, the background was green. When $A$ B D was presented, the background was red. Thus, the color cue was correlated with the stimulus set presented, but color was a redundant cue, since both $C$ and green were present when $C$ was correct in Serial Position 3 and both $D$ and red were present when D was correct in Serial Position 3.

A discrete-trials procedure with a between-trials correction contingency and a 2-sec intertrial interval was used in all conditions. At the beginning of a trial, the array was presented at the dim intensity on the bottom row of keys. The top row of keys was not used. Each correct selection of a stimulus caused the stimulus to change from the dim to the bright intensity for the duration of the trial.

The different possible permutations of the stimuli on the five keys were called arrays. For the one-stimulus condition, there were $S$ possible arrays, since the stimulus could be placed on each of the five keys. All 5 arrays were used in training. For strings with two or more stimuli, 10 arrays were chosen from the possible permutations. The 10 arrays were chosen so that each stimulus occurred on each key equally often, that is, so that stimuli and keys were not confounded.

After a correct trial, the array for the next trial was chosen from a randomized-blocks order of the 10 arrays, with 20 arrays per block. After an incorrect trial, the same array was presented again (a correction procedure). However, the subject had to start at the beginning of the string (Stimulus $A$ ) regardless of the location of the error on the prior trial. Thus, the subject had to complete correctly an array before being presented with the next array. This correction procedure prevented the use of an error to "skip over" a difficult array. Each session lasted for 140 correct trials or $45 \mathrm{~min}$ ( $60 \mathrm{~min}$ during the initial training), whichever came first.

Because the subjects made many errors during the initial sessions of the three-stimulus sequence, two procedures were used to facilitate acquisition. First, some shaping was employed by manually delivering food for components of the string. For example, pecks to A would be shaped for a subject that tended to peck $\mathrm{C}$ in the first serial position. Second, for four of the subjects, arrays that were more difficult were removed until the subject had mastered the easier arrays. When accuracy (number of correct strings/total number of strings) showed no systematic change over five sessions, the conditional string task was introduced.

In the conditional string task, the arrays contained all four stimuli (A, B, C, and D) and one dark key. Within each block of 20 correct trials, each of the 10 arrays was presented twice. Within a block, the background was green once and red once for each array, and the within-block order was determined randomly. The background color served as the conditional cue. When it was green, the correct order was A B C. When it was red, the correct order was A B D. This phase was terminated after 34 or 36 sessions.

Finally, the subjects were given two transfer tests. In each transfer test, 10 new arrays were used in place of the original arrays for 10 sessions. All other details were identical to the previous condition. 


\section{Results and Discussion}

Accuracy is defined as the number of correct strings divided by the sum of the number of correct and the number of incorrect strings. The chance level of accuracy of a three-stimulus string is the probability of randomly picking the correct key out of five available keys on three successive selections, which is .008 . A more conservative chance model would have the subjects randomly select from the dim keys only under the assumption that they had learned the bright-dim discrimination. This model would give a chance accuracy of $.167(1 / 3 \times 1 / 2 \times 1 / 1)$ for the three-stimulus string and $.042(1 / 4 \times 1 / 3 \times 1 / 2)$ for the conditional string. All of the accuracy levels presented below were clearly above chance.

Accuracy was $64.9 \%$ (range $52.4 \%$ to $75.3 \%$ ) on the last session of the three-stimulus string and dropped to $38.4 \%$ (range $27.7 \%$ to $75.3 \%$ ) on the first session of the conditional string. The drop was statistically significant $[F(1,7)=85.83]$. All statistical tests used the .05 level of significance. Training on the conditional string raised accuracy to $78.4 \%$ (range $72.5 \%$ to $84.8 \%$ ) on the session before the first transfer test.

The best measure of transfer uses only the first presentation of each new array on the two background colors, because responding to subsequent presentations may be influenced by the consequences of the responses to the prior presentations. The appropriate comparison for the first presentation accuracy on the new arrays is the accuracy for the first presentation on the old arrays within the previous session. This controls for possible warm-up effects.

The first exposure data for the first transfer showed a significant drop, $69.4 \%$ to $51.3 \%[F(1,7)=11.52]$, although the accuracy remained above chance. The second transfer test did not show a change in accuracy $[F(1,7)=2.71]$.

Introduction of the conditional string task produced a disruption of behavior followed by quick recovery. One possible explanation of the effect is that the subjects did not learn about color during the three-stimulus problem, that is, that they failed to associate. Colors were a redundant cue in the threestimulus problem, since the subject could learn to peck A B C when presented with those three stimuli and to peck A B D when presented with that stimulus set. Only when the conditional string was introduced did color become a relevant cue. The failure-ofassociation hypothesis would predict that the additional errors that occurred upon the introduction of the conditional string would be the incorrect choices of one of the variable stimuli. In order to test this hypothesis, an analysis of error types was performed.

In this task, there are five types of errors. Selections forward in the sequence (e.g., selecting $C$ first) are forward errors. Selections backward in the sequence (e.g., the sequence A B A) are backward errors. Selections of the same stimulus twice in succession (e.g., A B B) are repeat errors. Any selection of a dark key (a key not containing a stimulus) during a trial is a dark-key error. The fifth error type, the intrusion error, can occur only in a conditional string. This error is the mismatch of the variable stimulus and the background color, that is, D when the background is green and $C$ when the background is red. Since the number of opportunities to make different types of errors varies with error type, string length, serial position, etc., the probability of each error type was computed for each subject for each session.

For each session's data, the probability of an error type was computed by dividing the number of occurrences of the error type by the number of opportunities for that error type to occur (see Richardson \& Bittner, 1982, and Richardson \& Warzak, 1981, for more details). Figure 2 presents the mean error type probabilities for the last three sessions of the threestimulus problem, the first session of the conditional string, and the last three sessions of the conditional string. The error probabilities of the four error types present in both the three-stimulus problem and the conditional string showed little change from the last of the three-stimulus string to the first session of the conditional string. An analysis of variance of forward, backward, repeat, and dark-key errors for the end of the three-stimulus string and the first day of the conditional string showed no effect of condition $[F(1,7)<1]$, an effect of type of error $[F(3,21)=$ 118.29], and no interaction of condition and error type $[F(3,21)=1.64]$. The drop in accuracy at the beginning of the conditional string was due to the high probability of intrusion errors. By the end of the con-

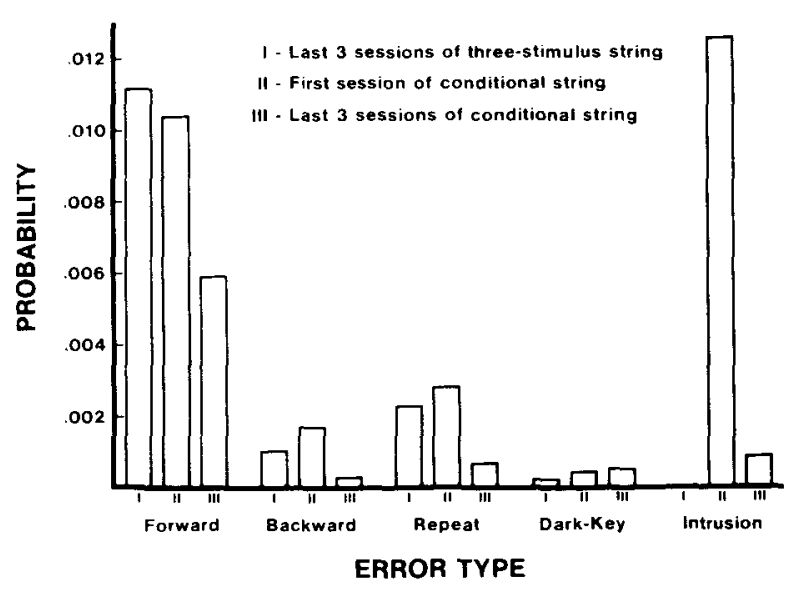

Figure 2. The probability for each type of error for the last sessions of the three-stimulus string, the first session of the conditional string, and the last sessions of the conditional string. 
ditional string training, the probability of an intrusion error dropped dramatically $[F(1,7)=110.56]$. Thus, under steady state conditions, both in the three-stimulus string and the conditional string, forward errors were much more probable than the other error types and the probabilities of the other error types were about equal.

Because the drop in accuracy following the introduction of the conditional string was accompanied by a high probability of intrusion errors but by no change in the probability of the other error types, the Eailure-of-association hypothesis was supported. The location of the intrusion errors is shown in Figure 3, which indicates the group mean probability of intrusion errors for each of the three serial positions for the first session of the conditional string divided into seven blocks of 20 correct trials. An analysis of variance showed significant effects of serial position $[F(2,14)=12,24]$, block $[F(6,42)=28.04]$, and serial position $\times$ block $[F(12,48)=2.90]$. The interaction was probably due to a floor effect, since the functions come together only when the functions of Serial Positions 2 and 3 cease to decrease at about .010 . There was a higher probability of intrusion errors in the third serial position, with the probability of intrusion errors dropping to approximately .010 by the end of the session. Thus, the probability of an intrusion error at the end of the first session of the conditional string was approximately the same as at the end of the conditional string training (see Figure 2). These data show that the subjects rapidly learned the color-lexigram association. This probably was due, in large part, to the use of color as the conditional cue. Pigeons learn color discriminations

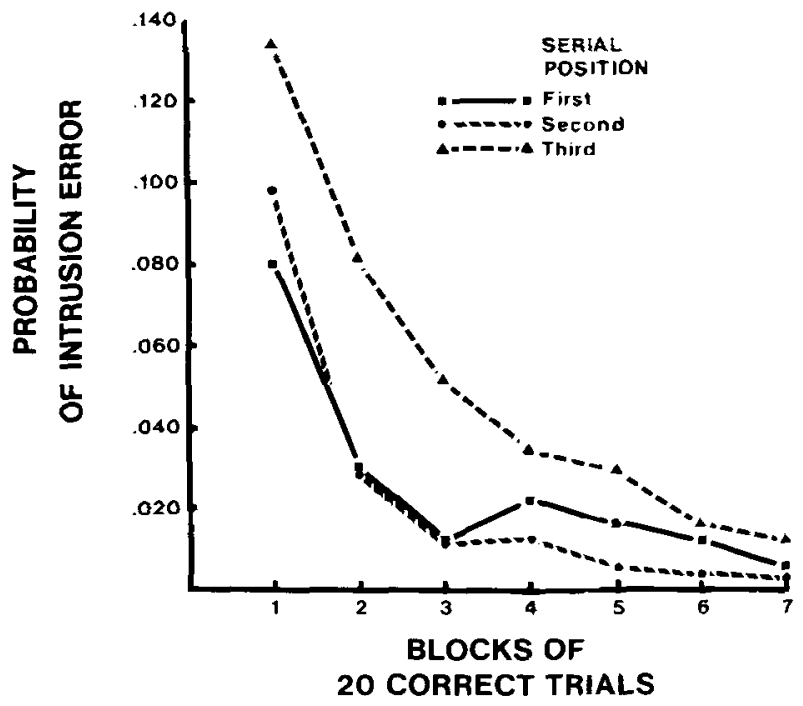

Figure 3. Probability of an intrusion error in each serial position as a function of blocks of $\mathbf{2 0}$ correct trials. very quickly. If a less salient conditional cue had been used, the transition from the three-stimulus string to the conditional string might have been much prolonged, if not impossible, without special training techniques.

The answer to both questions that Experiment 1 was designed to answer is yes. Pigeons car learn a stimulus string when the elements should not be especially salient, and pigeons can learn a conditional, symbolic, stimulus string.

In addition, the fact that the behavior transferred to new arrays limits the possible strategies for learning the string. Specifically, the transfer data showed that the birds were not responding to each different array as a different stimulus gestalt. For example, the array "A C D B" could be learned by a rule like "in the presence of this configuration, peck kays $1,5,2$, and 4 in that order." A set of 10 rules of this type would allow the subjects to perform well on the original arrays but would not allow for transfer to new arrays.

\section{EXPERIMENT 2}

In Experiment 1, the conditional cue was projected on each key containing a lexigram. We have viewed the arrays as consisting of four lexigrams on backgrounds of two different colors. The subjects might, however, view lexigram $A$ on red as being completely different from lexigram $A$ on green. If so, the subjects learned two different strings rather than a conditional string based on background color. That is, A B C D on green would be different stimuli from the same stimuli on red $\left(A^{\prime} B^{\prime} C^{\prime} D^{\prime}\right)$, so the subjects could learn to peck $A B C$, when available, and to peck $A^{\prime} B^{\prime} C^{\prime}$, when available. If this hypothesis is true, the subjects did not learn a conditional string.

In order to test this hypothesis, the background cue was removed from the lexigrams in Experiment 2. First, the background cue was removed from the variable stimuli only. Next, it was removed from all the lexigrams and placed on the key that did not contain a lexigram. If the subjects had learned the conditional string task as a conditional discrimination, they should have performed accurately regardless of the location of the conditional cue. In a final condition, the background cue was completely removed, so no conditional cue was presented.

\section{Method}

Subjects. The subjects from Experiment 1 were used in Experiment 2 . There was a period of approximately 1 month between the end of Experiment 1 and the beginning of Experiment 2.

Apparatus. Two chambers, identical to those of Experiment 1, were added to give a total of four chambers. Half the birds were switched to the new chambers.

Procedure. Subject 306 was changed from Stimulus Set B to Stimulus Set $\mathrm{A}$, and all birds were given 20 training sessions on 
the conditional string task used in Experiment 1. Next, the background cue was removed from lexigrams $C$ and $D$ of each array, so the fixed elements of the string, but not the variable elements, contained the conditional cue. After six sessions, the conditional cue was placed, once again, on all lexigrams. After 6 more sessions, the conditional cue was again removed from the variable elements for 4 additional sessions.

In the next condition, the background cue was removed from all lexigrams and was presented on the key that did not contain a lexigram. After 12 sessions under this condition, the background cue was removed from the no-lexigram key so that the display did not contain a conditional cue in any location (an empirical measure of chance accuracy for the conditional discrimination part of the task). After 4 sessions, the background cue was returned to the no-lexigram key for 10 sessions.

\section{Results and Discussion}

Accuracy for Sessions 16-62 is plotted in Figure 4. Statistical analyses of the changes in conditions were performed by comparing the last session of each condition with the first session of the following condition (using the .05 level of significance). All changes in conditions produced a statistically significant change in accuracy.

When the colors were first removed from the variable-lexigram keys, there was a large drop in accuracy $[F(1,7)=82.13]$, followed by recovery over four sessions. Replacing the colors on the variablelexigram keys also produced a small drop in accuracy $[F(1,7)=8.43]$, whereas removing the colors from the variable-lexigram keys for the second time was followed by a small increase in accuracy $[F(1,7)=7.15]$.

These data clearly show that the background color was not an integral or a necessary part of the variable-lexigram stimuli for the subjects; otherwise, they could not have performed well in the no-coloron-the-variable-lexigram-key condition. Evidently, the subjects saw the stimuli as lexigrams on a colored background, as did the experimenters.

The effect of removing the colors from the lexigram keys and placing them on the no-lexigram key was large, immediate, and lasting $[F(1,7)=439.84]$. There was some recovery during the first exposure to

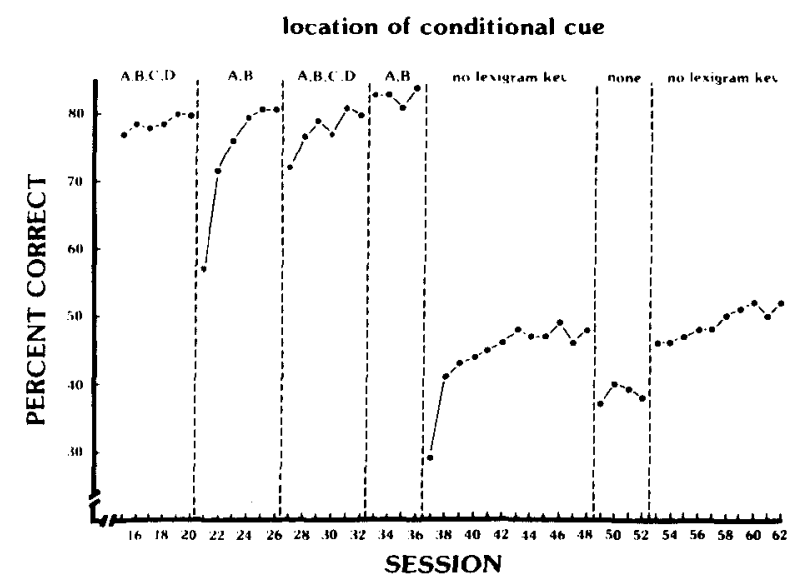

Figure 4. Accuracy during the conditions of Experiment 2. this condition, but accuracy never reached $50 \%$. The following condition had no colors present to serve as conditional cues. It was a conditional discrimination without conditional stimuli, an empirical measure of chance for the preceding condition. Note that this is a measure of chance for the conditional aspect of the task, not a total random-responding chance. The subjects still had the lexigrams to serve as discriminative stimuli for responses in Serial Positions 1 and 2. The absence of the conditional cue makes Serial Position 3 a coin toss (assuming selection of $\mathrm{C}$ or D). The expected value for accuracy would be .50 only if selections in Serial Positions 1 and 2 were always correct, which they were not. Comparison of the chance condition with the preceding $[F(1,7)=$ $17.70]$ and following $[F(1,7)=33.07]$ colors-on-theno-lexigram-key condition showed that the subjects were performing above chance when the colors were on the no-lexigram key. The last colors-on-the-nolexigram-key condition had an increase in accuracy between the first and last sessions $[F(1,7)=24.08]$. Improvement would probably have continued if training had been continued. Using a single eightstimulus string, we have found a slow increase in accuracy across 80 training sessions (Mitchell, Cole, \& Richardson, Note 1 ).

The low level of performance observed when the colors were not on the lexigrams was probably due to attentional factors. In matching-to-sample tasks, accuracy is typically very low unless the subject is required to peck, and thus observe, the sample prior to making the matching response (Eckerman, Lanson, \& Cumming, 1968). The high accuracy on the two conditions in which the colors were on some or all the lexigrams was probably due to the fact that the subjects had pecked, and thus observed, the conditional cue prior to choosing from the variable lexigrams. If a peck to the no-lexigram key had been required when it alone contained the colors, then an increase in accuracy might have been observed. Other ways of forcing attention to the conditional cues might also be effective. For example, the lexigrams could be lighted without a conditional cue, and any response could be counted as an error. After a variable time without a response, the conditional cue could be presented away from the lexigrams. The presence of the conditional cue would signal both the beginning of the trial and which variable lexigram would be correct. If the subjects have learned to attend to the conditional cue to avoid premature responses, then the cue should also control choice of the variable lexigram.

\section{GENERAL DISCUSSION}

The results of these studies show that the pigeons solved the two-string problem as a conditional discrimination, that is, that the selection of three of four stimuli came to be controlled by background 
color as the result of differential reinforcement of the strings in the presence of the two background colors. There are thus two types of discriminative behavior to be explained: the selection of the correct string and the selection of the correct sequence of items within a string. The selection of the correct string reduces, in the present experiments, to the selection of the correct item in Serial Position 3, the only variable position here. The selection of the correct item in Serial Position 3 could be due to simple paired-associate learning. The pigeon pecks $A$, then $B$, and then $C$ or $D$, depending on the state of the conditional stimulus (if green, peck C; if red, peck D). When the conditional color was on the lexigram keys, the pigeons had just pecked and thus observed the color while pecking A and B, which facilitated the subsequent selection of the correct lexigram in Serial Position 3. When the color was off the lexigram keys, the pigeons were not required to peck the conditional color prior to selecting $C$ or $D$ and thus may not have observed the color, which resulted in a lower level of accuracy.

Different explanations have been offered for the learning of the serial sequence in this type of paradigm. Straub, Seidenberg, Bever, and Terrace (1979) took the position that the pigeon learns a representation that allows it to keep its place while working through the required series of items. Richardson and Warzak (1981) suggested a chaining model, in which there are stimuli present at each point in the sequence that may act as discriminative stimuli for the next selection. For the present study, the chaining model would work as follows. Trial onset would produce discriminative stimuli controlling a high probability of the selection of $\mathbf{A}$; the selection of $A$ would produce stimuli controlling a high probability of the selection of $\mathrm{B}$; and the selection of $\mathrm{B}$ would produce stimuli that, in combination with the conditional color, would result in a high probability of the selection of $C$ or $D$, whichever was appropriate. The stimuli produced by a selection that serve as discriminative stimuli for the next selection could be external, internal, or both. In the present study, a likely candidate for part of the discriminative stimulus complex would be the bright state of the stimulus that had just been selected. Recall that the correct selection of a stimulus resulted in the change of that stimulus from the dim to the bright state. Although this feedback would be expected to be an important part of the discriminative stimulus, it is not necessary (Richardson \& Bittner, 1982; Straub \& Terrace, 1981), since distinctive natural consequences of pecking a specific stimulus would also be present. As would be expected if a salient part of the discriminative stimulus was removed, the Richardson and Bittner study showed lower accuracy for the condition in which the experimenter provided no feedback.

Straub and Terrace (1981) have demonstrated that pigeons trained to select a four-stimulus string, A B C D, can perform above chance on test trials consisting of an abbreviated form of the string, for example, A C or A D. Our chaining model can explain these data also.

In order to decide which responses will be predicted by the chaining model, we must consider how the discriminative stimuli come to control the next response, a question whose answer must consider the training procedure whereby the pigeons came to learn the task in the first place. The procedure is that the selection of $A$ in the presence of the discriminative stimuli existing after trial onset is followed by conditioned reinforcement and, after some within-chain delay, primary reinforcement when a correct sequence has been emitted. Our assumption is that the cues present after trial onset become strongly associated with the selection of $A$. In addition, these same cues will also be associated, to a lesser degree, with the selection of $B, C$, and $D$. These associations will occur to the degree that trial onset cues are also present when the later stimuli are correctly selected. There are two reasons that trial onset cues could be present when the later items have been correctly selected. First, since the successive selections occur over a short time period, the trial onset cues may not have completely faded from memory when the later selections occur. Second, the cues produced by the selection of $\mathrm{A}, \mathrm{B}, \mathrm{C}$, and $D$ may all have some elements in common with the cues produced by trial onset, a common-elements model of stimulus generalization. In general, there should be a gradient of association between the cues present at any point in the chain and all selections. Thus, after training with A B C D, the subject would select $A$ first on an $A D$ trial because trial onset cues are strongly associated with the selection of $\mathbf{A}$. The subject would then be likely to select $D$ next because the cues present after the selection of $A$ are associated with the selection of $D$ as well as with the selection of $B$ and $C$, which were not present on A D trials.

Straub and Terrace (1981) found that the pigeons performed well on the abbreviated strings and that in the case in which the abbreviated string consisted of $A$ and one other item, the accuracy increased with the number of items from the training string that were omitted from the two-stimulus test string [e.g., the A B test string had no items omitted, whereas the A D test string had two items (B and C) omitted]. This increase in accuracy seems counterintuitive and unpredictable from a chaining analysis. Why should accuracy be lower on A B test trials, in which the two items were in the same 
relative positions as in training, than on $A$ D test trials, which consisted of two items the pigeons had not been trained to peck successively? In order to understand how this distance effect is in fact predicted from our chaining model requires closer examination of the procedure used by Straub and Terrace (1981). That procedure did not have experimenterprovided feedback and did not count repeat pecks to a correctly selected item as errors; thus, when presented with the pair A B, the only possible way to make an overt error was to select $B$ in the first serial position. Failure to complete a trial in $15 \mathrm{sec}$ also terminated the trial and was in one sense an error. This time limit was seldom reached, and, since it was not relevant to the present analysis, it was ignored. If $A$ was selected first, then the trial could not end in an error, since subsequent selections of $A$ were not counted as errors and selection of B, the only other stimulus present, would result in a correct trial. Likewise, when the pair A D had been presented, the only possible error was the selection of $D$ in the first serial position.

Now consider what happened on two-item test trials. First, since trial onset consisted of presenting only two of the four cues used during training, the abbreviated string trial onset cues could differ slightly from the regular training trial onset cues. However, there is no reason to expect this difference in trial onset cues to produce a bias for any test item. When Items $A$ and $B$ are presented as an abbreviated string, both should be strongly associated with the trial onset cues, but Item A should be more strongly associated. At the other extreme, with the presentation of Items $A$ and D as an abbreviated string, Item $D$ should be less strongly associated with the trial onset cues than Item B on the A B trials, due to the gradient of association suggested above. Thus, a strongly associated item (B) would compete more effectively with Item A on A B trials, whereas a weakly associated item (D) would compete less effectively with Item A on A D trials. The weaker competition on A D trials would result in a higher probability of selecting $A$ in Serial Position 1 , and, as we have shown, once $A$ has been selected, the only other selection that would count is the selection of $D$, resulting in a correct string. Repeat selections of $A$ are not counted as errors. The higher probability of selecting $A$ in the first serial position on A D trials results in a higher accuracy for A D trials. The same reasoning would predict the accuracy on A C trials to be between the accuracy on A B and A D trials. Thus, these results of the abbreviated string tests are predicted by the chaining model. If repeat selections had been counted as errors in the Straub and Terrace (1981) study, the results might have been different, since the pigeons may have been more likely to repeatedly peck $A$ on $A$ D trials, on which $D$ weakly competes with $A$, than on A B trials, on which B strongly competes with A.

Finally, Thompson and Church (1980) presented an analysis of the language-like behavior of Lana Chimpanzee, which may be relevant to the present paradigm. Lana Chimpanzee (Rumbaugh, 1977) learned to select a string of symbols depending on the conditional cues present in the environment. For example, if the three conditional cues (1) object is present, (2) object is food, and (3) object is in machine were present in the environment, then Lana could select the stock sentence "Please machine give (incentive)." If Lana inserted the lexigram for the food that was visible in the machine into the place of (incentive), then she would receive the food in the machine. We feel that the paradigm used with Lana is similar to the conditional stringing paradigm. In fact, the work with Lana provided the impetus for the present work. The Thompson and Church (1980) analysis ascribed Lana's selection of a stock sentence (stimulus string) to conditional discrimination learning, while paired associative learning was responsible for the selection of the appropriate lexigram to represent an incentive in the stock sentence. This analysis would work equally well for the behavior observed in the present study.

\section{REFERENCE NOTE}

1. Mitchell, Z. P., Cole, M. A., \& Richardson, W. K. The origin of forward errors in serial lists by pigeons. Paper presented at the meeting of the Southeastern Psychological Association, Atlanta, March 1981.

\section{REFERENCES}

Capaldi, E. J., Verry, D. R., \& Davidson, T. L. Memory, serial anticipation pattern learning, and transfer in rats. Animal Learning \& Behavior, 1980, 8, 575-585.

Eckerman, D. A., Lanson, R. N., \& Cummina, W. W. Acquisition and maintenance of matching without a required observing response. Journal of the Experimental A nalysis of Behavior, $1968,11,435-441$.

HuLse, S. H. Cognitive structure and serial pattern learning by animals. In S. H. Hulse, H. Fowler, \& W. K. Honig (Eds.), Cognitive processes in animal behavior. Hillsdale, N.J: Erlbaum, 1978.

Outon, D. S. Characteristics of spatial memory. In S. H. Hulse, H. Fowler, \& W. K. Honig (Eds.), Cognitive processes in animal behavior. Hillsdale, N.J: Erlbaum, 1978.

Richandson, W. K., \& BitTwen, B. J. Stimulus stringing by pigeons: Effect of feedback for correct selections. Animal Learning \& Behavior, 1982, 10, 35-38.

Richardson, W. K., \& Warzak, W. J. Stimulus stringing by pigeons. Journal of the Experimental Analysis of Behavior, 1981, 36, 267-276.

RUMBaUar, D. (Ed.). Language learning by a chimpanzee: The Lana project. New York: Academic Press, 1977.

Sand8, S. F., \& Wrioht, A. A. Serial probe recognition performance by a rhesus monkey and a human with 10- and 20-item 
lists. Journal of Experimental Psychology: Animal Behavior Processes, 1980, 6, 386-396.

Straub, R. O., Seidenderg, M. S., Bever, T. G., \& Terrace, H. S. Serial learning in the pigeon. Journal of the Experimental Analysis of Behavior, 1979, 32, 137-148.

Straub, R. O., \& Terrace, H. S. Generalization of serial learning in the pigeon. Animal Learning \& Behavior, 1981, 9, 454-468.

Thompson, R. T., \& Church, R. M. An explanation of the language of a chimpanzee. Science, 1980, 208, 313-314. von Glasersfeld, E. Linguistic communication: Theory and definition. In D. M. Rumbaugh (Ed.), Language learning by a chimpanzee: The Lana project. New York: Academic Press, 1977.

(Manuscript received April 26, 1982;

revision accepted for publication August 29, 1982.) 